\title{
Causation, Association and Confirmation
}

\author{
Gregory Wheeler ${ }^{1} \&$ Richard Scheines ${ }^{2}$
}

\begin{abstract}
Many philosophers of science have argued that a set of evidence that is "coherent" confirms a hypothesis which explains such coherence. In this paper, we examine the relationships between probabilistic models of all three of these concepts: coherence, confirmation, and explanation. For coherence, we consider Shogenji's measure of association (deviation from independence). For confirmation, we consider several measures in the literature, and for explanation, we turn to Causal Bayes Nets and resort to causal structure and its constraint on probability. All else equal, we show that focused correlation, which is the ratio of the coherence of evidence and the coherence of the evidence conditional on a hypothesis, tracks confirmation. We then show that the causal structure of the evidence and hypothesis can put strong constraints on how coherence in the evidence does or does not translate into confirmation of the hypothesis.
\end{abstract}

\section{Introduction}

It has long been held that "coherent" evidence confirms a hypothesis which explains the coherence of such evidence. For example, if five of your friends come down with a stomach ailment on Tuesday after going to the same restaurant on Monday evening without even seeing each other, then this powerfully confirms the hypothesis that they got food poisoning from that restaurant. The hypothesis explains the evidence because the food served by the restaurant is a common cause of the five separate stomach ailments. Alternatively, if we know that the five friends did not eat at the same restaurant on Monday, but we do know that all met each other and either shook hands or kissed at one time or another during the day, then the co-occurrence of their Tuesday stomach trouble is no evidence at all for restaurant induced food poisoning; the explanation is quite different: they passed a stomach virus from one to the other. The difference between the cases is not the "coherence" of the evidence, but rather the causal explanation of this coherence. In the first case the association of their stomach ailment is due entirely to the influence of a common cause, and in the second case it is due entirely to a causal relationship directly between the friends.

In this paper we discuss more systematically and formally the connections between 1) the causal relationships between a hypothesis under consideration and evidence relevant to the hypothesis, 2) the coherence of this evidence, and 3) the degree to which the evidence confirms the hypothesis. 
Bayesian epistemologists have given us probabilistic models of both coherence and confirmation, and philosophers, computer scientists, and statisticians have given us probabilistic models of causal systems. ${ }^{3}$ Our task is to show how these pieces might or might not fit together. In this paper, our focus is not on coherence, confirmation, or how they are to be modeled, but rather on the relationship between coherence and confirmation and how this relationship might be mediated by the causal relationships among the hypothesis and the evidence. In particular, we will consider situations in which the pieces of evidence in two sets of evidence are equally relevant to a hypothesis individually, the coherence of the sets differ, but the causal structure is identical, as well as situations in which the pieces are equally relevant, the sets are equally coherent, but the causal structure is different. These cases will hopefully bring out the role that causal structure plays in mediating the relationship between coherence and confirmation.

The organization of the paper is as follows. In section 2 we review coherentism from the perspective of Bayesian epistemology. In section 3 we introduce the assumptions and models we will use to give structure to notions of coherence and confirmation. In section 4 we discuss the relationship between coherence and confirmation simpliciter. In section 5 we introduce the idea of focused correlation (Wheeler 2009) and extend results connecting coherence to confirmation through focused correlation. In section 6 we present the case for making causal beliefs explicit, and trace several consequences for the relationship between coherence and confirmation that arise solely from the causal structure governing the evidence and hypothesis.

\section{Coherence as Probabilisitic Association}

In 1985, Laurence BonJour ${ }^{4}$ gave some structure to the coherence theory of justification by laying out criteria for a theory of coherence. (He later despaired of meeting those demands and quit the theory altogether, but that is another story. ${ }^{5}$ ) His postulates for coherentism mention a role for probability, and several authors, operating under the heading of "Bayesian epistemology", have explored the prospects of developing a probabilistic measure of coherence along his basic outline. ${ }^{6}$

Much of this work in Bayesian epistemology concerns coherence among a set of statements and whether a probabilistic measure of coherence can be adduced which is "truth-conducive", that is, whether in fact higher coherence among a set means more reason for belief in that set, ceteris paribus. Another perspective divides things up into hypotheses we care about, either decision theoretically or scientifically, and evidence we might collect, possess, or already know about which may be epistemically relevant to these hypotheses. Coherence in this setting is coherence among only the evidence, and the question explored is whether evidential coherence makes any epistemic difference to the hypotheses upon which that evidence might bear, ceteris paribus. It is this perspective that we explore here. 
Perhaps the most obvious and most common account of epistemic coherence is to portray coherence as probabilistic association, that is, the degree to which evidential propositions co-occur relative to how much they might or might not. We take binary evidential variables as representing propositions. For example, suppose that $E_{l}$ is a binary evidence variable representing a witness report, where $E_{l}=$ true codes for "the witness reported fact 1 is the case," written $E_{1}$ for short, and $E_{l}=$ false codes for "the witness reports that fact 1 is not the case," written $\neg \mathrm{E}_{1}$ for short. Then perhaps the most straightforward account of evidential coherence in this tradition ${ }^{7}$ is the association (i.e., deviation from independence) measure advanced by Tomoji Shogenji (1999), which is presented here in standard and conditional form: ${ }^{8}$

$$
S\left(E_{1}, E_{2}\right)=\frac{P\left(E_{1} \cap E_{2}\right)}{P\left(E_{1}\right) P\left(E_{2}\right)} \quad S\left(E_{1}, E_{2} \mid H\right)=\frac{P\left(E_{1} \cap E_{2} \mid H\right)}{P\left(E_{1} \mid H\right) P\left(E_{2} \mid H\right)} .
$$

Here binary evidence variables $E_{1}$ and $E_{2}$ are positively associated (associated conditional on a hypothesis, $H$ ) if $S$ is greater than 1, independent (conditionally independent on $H$ ) if $S$ is 1 , and negatively associated (negatively associated conditional on $H$ ) if $S$ is less than 1 .

Shogenji interprets $S$ (in unconditional form) as a measure of "pairwise coherence", which evaluates "the degree by which the two beliefs $\left[E_{1}=\right.$ true and $E_{2}=$ true $]$ are more likely true together than they would be if they were [probabilistically independent]" (Shogenji 1999, 339). Even though Shogenji interprets this measure to apply to cases in which the evidence variables are assigned "true", and thus to concern the coherence of a pair of propositions, the notion of coherence here is probabilistic association among evidence variables rather than concurrent agreement of an information set. After all, the measure has no meaning without a joint probability distribution over all the possible states for the variables.

Association fails to capture many aspects of evidential coherence, and some might argue that it fails to capture any of them. Our interest is not in entering that debate, however, it is rather in considering the relationship between coherence and confirmation, and how causation helps mediate this relationship. So, without prejudicing the debate or claiming it is adequate, we will use Shogenji's measure of coherence in what follows.

\section{Confirmation}

The debate about how to characterize the idea (or mirage) of confirmation, and whether the probability calculus is the right tool for the job, is similarly contentious and far from settled. We have no desire to enter this fray either - only to examine how popular probabilistic conceptions of confirmation relate to popular probabilistic notions of coherence. 
Several measures of confirmation have been offered. A few of the more popular ones use probability to express how much confirmation an evidence set $\mathbf{E}$ provides to a hypothesis $H$ (Elles and Fitelson 2002):

- $\quad r(\mathrm{H}, \mathbf{E})=_{d f} \log \frac{P(\mathrm{H} \mid \mathbf{E})}{P(\mathrm{H})}$

- $l(H, \mathbf{E})={ }_{d f} \log \frac{P(\mathbf{E} \mid \mathrm{H})}{P(\mathbf{E} \mid \neg \mathrm{H})}$

- $k o(\mathrm{H}, \mathbf{E})=d_{d f} \frac{P(\mathbf{E} \mid \mathrm{H})-P(\mathbf{E} \mid \neg \mathrm{H})}{P(\mathbf{E} \mid \mathrm{H})+P(\mathbf{E} \mid \neg \mathrm{H})}$

Cohen (1977) and Earman (1992) define the incremental confirmation of $\mathrm{E}_{2}$ on $\mathrm{H}$ after we already know $\mathrm{E}_{1}$ :

$$
\operatorname{inc}_{1}\left(\mathrm{H}, \mathrm{E}_{1}, \mathrm{E}_{2}\right)=_{d f} P\left(\mathrm{H} \mid \mathrm{E}_{1} \cap \mathrm{E}_{2}\right)-P\left(\mathrm{H} \mid \mathrm{E}_{1}\right)
$$

An extension of incremental confirmation that normalizes for how much room above $\mathrm{P}\left(\mathrm{H} \mid \mathrm{E}_{1}\right)$ there is for $\mathrm{E}_{2}$ to "boost" the posterior of $\mathrm{H}$ is:

$$
i n c_{2}\left(\mathrm{H}, \mathrm{E}_{1}, \mathrm{E}_{2}\right)==_{d f} \frac{P\left(\mathrm{H} \mid \mathrm{E}_{1} \cap \mathrm{E}_{2}\right)-P\left(\mathrm{H}_{\mid} \mathrm{E}_{1}\right)}{1-P\left(\mathrm{H}_{1} \mathrm{E}_{1}\right)}
$$

\section{Confirmation and Coherence}

Using Shogenji's measure of association as a measure of evidential coherence, and any of the measures of confirmation given above, we can ask whether there is a relationship between the coherence of an evidence set and the confirmation it provides to a hypothesis. More formally, is it the case, or, under what conditions is it the case, that for two evidence sets $\mathbf{E}$ and $\mathbf{E}$ ', and one of the measures of confirmation above, conf,

$$
\mathrm{S}(\mathbf{E})>\mathrm{S}\left(\mathbf{E}^{\prime}\right) \Rightarrow \operatorname{conf}_{\mathrm{i}}(\mathrm{H}, \mathbf{E})>\operatorname{conf}_{\mathrm{i}}\left(\mathrm{H}, \mathbf{E}^{\prime}\right) \text { ? }
$$

As many authors have noted, the answer is clearly no. It is not the coherence of the evidence, but its relation to $\mathrm{H}$ and the reason for the coherence that makes the difference. For example, consider again the food poisoning vs. communicable virus hypotheses and the evidence that five of your friends are stricken with stomach problems on Tuesday. Whatever the coherence of this evidence in Shogenji's sense, it provides quite different confirmation to each hypothesis. The problem is that it is not the coherence or lack of such between the health of our five friends, but the reason for the coherence that is telling. 
Probabilistic attempts to secure a connection between coherence and confirmation either smuggle in a reason for the coherence (e.g., the partially reliable witness model of Hartmann and Bovens, 2003a,b), or rely upon a definition of coherence that is partially built from the confirmation relation (e.g., Bovens and Hartmann, 2003b).

\section{Ceterus Paribus}

What we now want to explore is whether, all else equal, evidence sets that are differentially coherent provide differential confirmation to a hypothesis $\mathrm{H}$. In this section we attempt to formalize and clarify "all else equal."

In what follows, we will assume that the domain $\mathbf{D}=<\mathrm{H}, \mathbf{E}>$ is a hypothesis $H=$ true and an evidence set $\mathbf{E}=\left\{E_{1}=\right.$ true,,.,$E_{\mathrm{n}}=$ true), where $H$ and $E_{1}, . ., E_{\mathrm{n}}$ are propositional (binary) variables, none of which are logically related. A proposition conveniently expresses either the content of a proposition, or a witness report of a proposition. Extending what follows to real-valued variables is certainly possible.

By insisting that no logical relations obtain, which we do for two reasons, we mean that there are positive probability distributions over $\mathbf{D}$ in which every pair of variables $X$ and $Y$ are probabilistically independent. This is not possible, for example, in a setting in which $\mathrm{E}_{1}=$ The Butler did it, and $\mathrm{E}_{2}=$ The Butler did it or The Cook did it, for in no positive distribution is $E_{1}$ independent of $E_{2}$. We assume this in order to activate the theory of causal Bayes nets, which require variables that are unrelated logically, and also to avoid what we take to be distracting puzzles that trade on logical relations between variables. $^{9}$

We assume that $P(\mathbf{D})$, a probability distribution over a domain of propositions $\mathbf{D}=$ $<\mathrm{H}, \mathbf{E}>^{10}$ is positive.

We say that two distinct pieces of evidence $E_{i}$ and $E_{j}$ are equally confirmatory for a hypothesis $\mathrm{H}$ iff

- $P\left(\mathrm{H} \mid \mathrm{E}_{\mathrm{i}}\right)=P\left(\mathrm{H} \mid \mathrm{E}_{\mathrm{j}}\right)$, and

- $P\left(\mathrm{H} \mid \neg \mathrm{E}_{\mathrm{i}}\right)=P\left(\mathrm{H} \mid \neg \mathrm{E}_{\mathrm{j}}\right)$

We consider two assumptions

(A1) Positive Relevance: all propositions in a set of evidence $\mathbf{E}$ are positively relevant to $\mathrm{H}$, i.e., $\forall \mathrm{E}_{\mathrm{i}} \in \mathbf{E}, P\left(\mathrm{H} \mid \mathrm{E}_{\mathrm{i}}\right)>P(\mathrm{H})>P\left(\mathrm{H} \mid \neg \mathrm{E}_{\mathrm{i}}\right)$

(A2) Equal Relevance: all propositions in a set of evidence $\mathbf{E}$ are equally confirmatory, i.e., $\forall \mathrm{E}_{\mathrm{i}} \mathrm{E}_{\mathrm{j}} \in \mathbf{E}, P\left(\mathrm{H} \mid \mathrm{E}_{\mathrm{i}}\right)=P\left(\mathrm{H} \mid \mathrm{E}_{\mathrm{j}}\right), P\left(\mathrm{H} \mid \neg \mathrm{E}_{\mathrm{i}}\right)=P\left(\mathrm{H} \mid \neg \mathrm{E}_{\mathrm{j}}\right)$.

Call an evidence set whose elements satisfy (A1) with respect to $\mathrm{H}$ a positive 
evidence set for $\mathrm{H}$, and call an evidence set whose elements satisfy (A1) and (A2) for H an equally positive evidence set (epe) for $H$.

These assumptions probabilistically encode what we take "ceteris paribus" to mean in an investigation on the relation between probabilistic notions of coherence and confirmation. We want to compare the confirmatory power for $\mathrm{H}$ of two sets of evidence $\mathbf{E}$ and $\mathbf{E}$ ', where $\mathbf{E}$ and $\mathbf{E}$ ' are identical in all respects except for their coherence. That is, we want to consider sets $\mathbf{E}$ and $\mathbf{E}$ ' which are comparable in terms of how confirmatory each individual piece of evidence is to $\mathrm{H}$, but that differ in terms of their overall coherence, and ask which set does more to confirm $\mathrm{H}$. This is how we interpret the question: Does more coherent evidence do more to confirm $\mathrm{H}$, ceteris paribus.

\section{Focused Correlation}

Wheeler (2009) attempted to address the apparent disconnect between coherence and confirmation by using a relation between coherence and conditional coherence on a hypothesis first introduced by Myrvold (1996). Wheeler examined how different degrees of "focused correlation" track different degrees of confirmation.

The focused correlation of a set of evidence $\mathbf{E}=\left\{\mathrm{E}_{1}, . ., \mathrm{E}_{\mathrm{n}}\right)$ with respect to a hypothesis $\mathrm{H}$ is the ratio of the coherence/association of the evidence conditional on $\mathrm{H}$ to the coherence/association of the evidence simpliciter, which can be expressed generally as:

$$
\operatorname{For}_{\mathrm{H}}\left(E_{1}, . ., E_{n}\right):=\frac{S\left(E_{1}, . ., E_{n} \mid H\right)}{S\left(E_{1}, . ., E_{n}\right)}=\frac{\frac{P\left(E_{1}, . ., E_{2} \mid H\right)}{P\left(E_{1} \mid H\right), . ., P\left(E_{n} \mid H\right)}}{\frac{P\left(E_{1}, . ., E_{n}\right)}{P\left(E_{1}\right), . ., P\left(E_{n}\right)}}=\frac{P\left(H \mid E_{1}, . ., E_{n}\right) P(H)^{n-1}}{P\left(H \mid E_{1}\right), . ., P\left(H \mid E_{n}\right)}
$$

Wheeler (2009) drew several connections between focused correlation and confirmation. Before examining the role of causal structure, we strengthen these connections for the case of evidence sets with two variables.

Consider Hypothesis $H$ and evidence sets $\mathbf{E}=\left\{\mathrm{E}_{1}, \mathrm{E}_{2}\right\}$ and $\mathbf{E}^{\prime}=\left\{\mathrm{E}_{1}, \mathrm{E}_{3}\right\}$ satisfying assumption (A1) above. For each of the confirmation measures above, the confirmation of $\mathrm{H}$ on an evidence set $\mathbf{E}$ is positive (greater than 0 ) if $\operatorname{For}_{\mathrm{H}}(\mathbf{E})$ is positive (greater than $1)$. 
Proposition 1: If $\mathbf{E}$ is a positive evidence set for $\mathrm{H}$, and $\operatorname{For}_{\mathrm{H}}(\mathbf{E})>1$, then all of the following hold:

- $r(\mathrm{H}, \mathbf{E})>0$

- $l(\mathrm{H}, \mathbf{E})>0$

- $k o(\mathrm{H}, \mathbf{E})>0$

- $\quad \operatorname{inc}_{1}(\mathrm{H}, \mathbf{E})>0$

- $\quad \operatorname{inc}_{2}(\mathrm{H}, \mathbf{E})>0$

Proposition 1 says that for any evidence set $\mathbf{E}$ that satisfies (A1), if $\mathbf{E}$ has a focused correlation for $\mathrm{H}$ above 1, then $\mathbf{E}$ provides positive confirmation of $\mathrm{H}$ by any of the above confirmation measures. If a set of evidence has more Shogenji coherence conditional on $\mathrm{H}$ that it does unconditionally, then the evidence provides positive confirmation to $\mathrm{H}$. This is good progress, but because the condition is not necessary, it still leaves open the situations in which $\operatorname{For}_{H}(\mathrm{E})<1$.

When we strengthen the assumptions regarding the uniformity of the evidence, focused correlation tracks confirmation:

Proposition 2: If $\mathbf{E}=\left\{\mathrm{E}_{1}, \mathrm{E}_{2}\right\}$ and $\mathbf{E}^{\prime}=\left\{\mathrm{E}_{1}, \mathrm{E}_{3}\right\}$, and $\mathbf{E} \cup \mathbf{E}^{\prime}$ is an equally positive evidence set for $\mathrm{H}$, then all of the following inequalities are equivalent:

- $\operatorname{For}_{\mathrm{H}}(\mathbf{E})>\operatorname{For}_{\mathrm{H}}\left(\mathbf{E}^{\prime}\right)$

- $r(\mathrm{H}, \mathbf{E})>r(\mathrm{H}, \mathbf{E} ')$

- $l(\mathrm{H}, \mathbf{E})>l(\mathrm{H}, \mathbf{E} ')$

- $k o(\mathrm{H}, \mathbf{E})>k o\left(H, \mathbf{E}^{\prime}\right)$

- $\quad \operatorname{inc}_{1}(\mathrm{H}, \mathbf{E})>\operatorname{inc} 1(\mathrm{H}, \mathbf{E} ')$

- $\quad i n c_{2}(\mathrm{H}, \mathbf{E})>\operatorname{inc} 2(\mathrm{H}, \mathbf{E} ')$

So in several senses, focused correlation tracks confirmation and incremental confirmation, whereas simple coherence (association) does not. Looking at the formula for focused correlation, it is immediate that two equally positive evidence (epe) sets can have equal association while having unequal focused correlation, and thus confirmation.

Why does the focused correlation capture something about the relationship between coherence and confirmation? Part of the answer, we believe, is the causal structure governing the system.

\section{Causal Structure}

Generally, Causal Bayes Nets (CBNs) provide all the apparatus needed to represent causal structure, ${ }^{11}$ and to characterize the constraints such structures impose on the probability distributions they might represent. Let a causal graph $\mathbf{G}=\{\boldsymbol{V}, \mathbf{E}\}$ be a set of random variables $\boldsymbol{V}$ and a set of directed edges $\mathbf{E}$ such that $E_{\mathrm{i}} \rightarrow E_{\mathrm{j}} \in \mathbf{E}$ if and only if $E_{\mathrm{i}}$ 
is a direct cause of $E_{\mathrm{j}}$ relative to $\boldsymbol{V}$. The set of direct causes of a variable are its parents. A set of variables $\boldsymbol{V}$ is causally sufficient just in case for every pair of variables $V_{\mathrm{i}}, V_{\mathrm{j}} \in$ $\boldsymbol{V}$, the common causes of $V_{\mathrm{i}}, V_{\mathrm{j}}$ are also in $\boldsymbol{V}$.

An acyclic causal graph $\mathbf{G}$ and a probability distribution $P(\boldsymbol{V})$ over the variables ${ }^{12}$ in $\mathbf{G}$ satisfy the Causal Markov Axiom (Spirtes, Glymour and Scheines, 2000) just in case $P(\boldsymbol{V})$ factors according to the causal graph:

$$
\mathrm{P}(\mathrm{V})=\prod_{X \in J} P(X \mid \text { parents }(X))
$$

This factorization ${ }^{13}$ imposes independence constraints on the distributions $P(\boldsymbol{V})$ which can be generated by a given causal graph. These constraints are characterized by the graph-theoretic relation of $d$-separation, (Pearl, 1988). One can view the independence constraints imposed by this factorization as the non-parametric consequences of qualitative causal structure.

An additional axiom typically applied to CBNs is the Faithfulness assumption (Spirtes, et al., 2000). A graph $\mathbf{G}$ and a probability distribution $P(\boldsymbol{V})$ over the variables ${ }^{14}$ in $\mathbf{G}$ satisfy the Faithfulness Axiom just in case the only independence relations in $P(\boldsymbol{V})$ are those entailed by the Causal Markov axiom (d-separation).

If causal structure alone plays a mediating role between coherence and confirmation, then it should be through the independence constraints in distributions that are Markov and Faithful to the causal graphs that govern the relationship between evidence and hypothesis.

One obvious interpretation of the Bovens and Hartmann 2003 model of partially reliable, independent witness reports is Figure 1. 


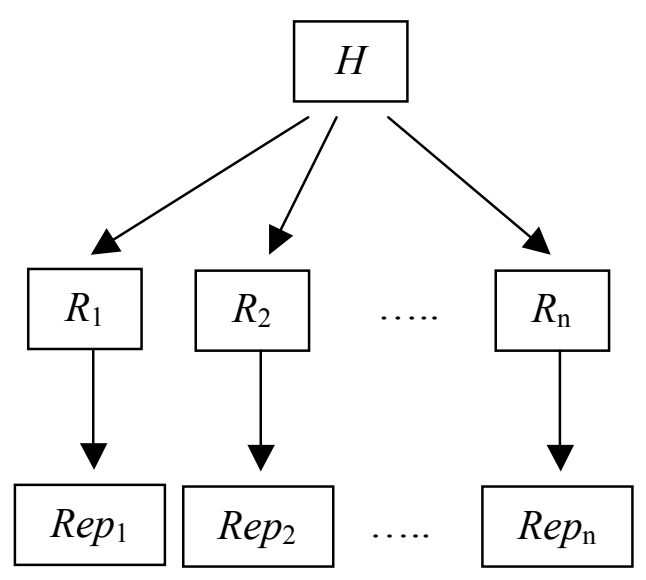

Figure 1: Common Cause Model for Bovens and Hartmann

A simplification of the Bovens-Hartmann model is the single-factor common cause model (Figure 2).

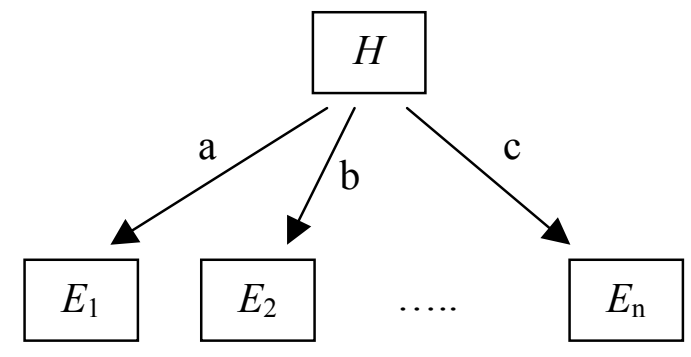

Figure 2: Single-Factor Common Cause Model

Interpreted as a CBN this model entails the evidence is independent conditional on $\mathrm{H}$, that is, any coherence among the evidence is due to $\mathrm{H}$ :

$$
\forall \mathrm{i}, \mathrm{j}, E_{\mathrm{i}} \_\|_{-} E_{\mathrm{j}} \mid H .^{15}
$$

How does the causal structure in the single-factor common cause model mediate the relationship between coherence and confirmation? The answer is that, in this model, the coherence of the evidence is strictly a function of the degree to which the evidence is individually associated with the hypothesis, so there is no way to separate coherence and confirmation - they are perfectly intertwined. ${ }^{16}$

How does causal structure more generally constrain or mediate the relationship between coherence and confirmation? Consider a causal model (Figure 3) that simultaneously represents three important extremal cases: 
1. All the coherence among the evidence is because of the hypothesis $\left(\mathbf{E}=\left\{\mathrm{E}_{1}, \mathrm{E}_{2}\right\}\right)$

2. None of the coherence among the evidence is because of the hypothesis $\left(\mathbf{E}^{\prime}=\left\{\mathrm{E}_{1}, \mathrm{E}_{3}\right\}\right)$

3. The evidence has no coherence, but is individually related to the hypothesis (E', $\left.=\left\{\mathrm{E}_{1}, \mathrm{E}_{4}\right\}\right)$

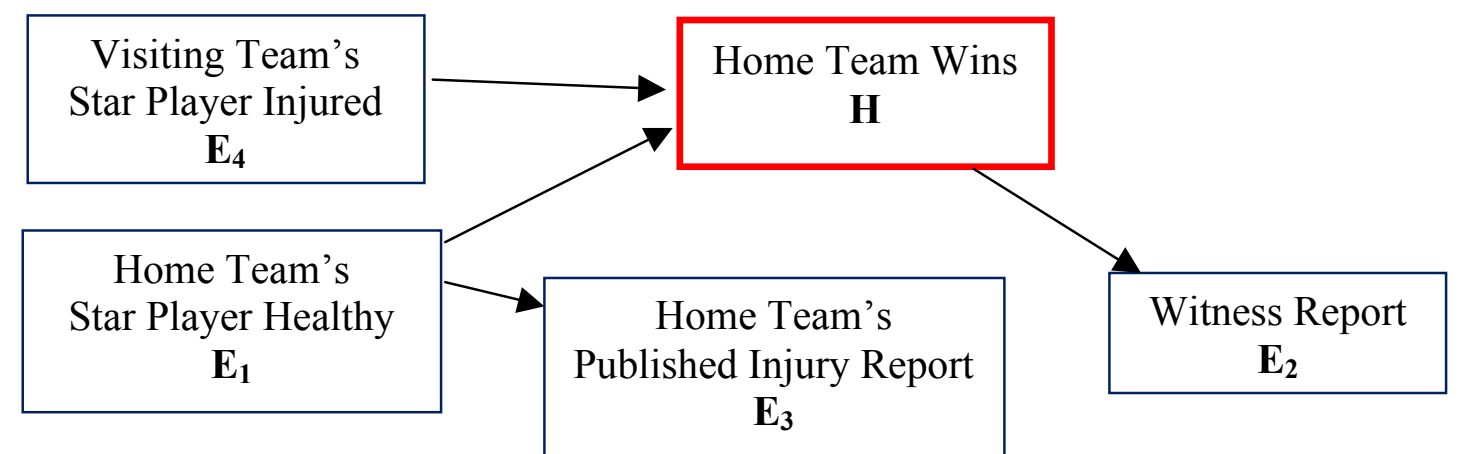

Figure 3: Causal Model of Home Team Wins

The hypothesis H of interest is whether one's home sporting team wins in some big game that you cannot directly witness. Consider several pieces of evidence relevant to this hypothesis.

$E_{1}$ is whether or not the home team's star player is injured going into the game, and similarly for $E_{4}$ and the visiting team's star player. We "code" $E_{1}$ as health and $E_{4}$ as "injury" so that both are positively relevant to $\mathrm{H}$. Both of these facts are evidence of, but also causes of, the hypothesis of interest. As the star players for the two teams have no causal connection relating to injury, we assume that $E_{1}$ and $E_{4}$ are causally and probabilistically independent. $\mathrm{E}_{3}$ is the published injury report about the home team's star player prior to the game. As any sports fan knows, such reports are only partially reliable. ${ }^{17}$ Still, we assume that such a report is an effect of whether or not the home team's star player is in fact healthy, and probabilistically independent of everything else given the state of the star player's health. Finally, we have the testimony of a partially reliable witness $\mathrm{E}_{2}$ on the outcome of the game.

First, consider the two sets of evidence $\mathbf{E}=\left\{\mathrm{E}_{1}, \mathrm{E}_{2}\right\}$ and $\mathbf{E}^{\prime}=\left\{\mathrm{E}_{1}, \mathrm{E}_{3}\right\}$. The coherence in $\mathbf{E}$ is for the same reason that different effects of a common cause are coherent: any coherence between $E_{1}$ and $E_{2}$ is the result of the correlation between $E_{1}$ and $H$ and between $\mathrm{H}$ and $\mathrm{E}_{2}$. $\mathbf{E}^{\prime}$ is the other extreme - none of the coherence between $\mathrm{E}_{1}$ and $\mathrm{E}_{3}$ is the result of the correlation between $\mathrm{E}_{1}$ and $\mathrm{H}$ and between $\mathrm{H}$ and $\mathrm{E}_{3}$. If $\mathbf{E}$ and $\mathbf{E}$ ' have 
identical coherence, do they afford different degrees of confirmation to H? Since both sets share $E_{1}$, this reduces to the question of whether the incremental confirmation afforded by $E_{2}$ always exceeds that of $E_{3}$, or vice versa, or neither one.

By the causal structure of this model, $\mathrm{H}$ and $\mathrm{E}_{3}$ are independent conditional on $\mathrm{E}_{1}, P(\mathrm{H} \mid$ $\left.\mathrm{E}_{1}\right)=P\left(\mathrm{H} \mid \mathrm{E}_{1}, \mathrm{E}_{3}\right)$, thus $\mathrm{E}_{3}$ provides zero incremental confirmation after $\mathrm{E}_{1}$. Thus the question of whether $\mathbf{E}$ and $\mathbf{E}$ ' afford different degrees of confirmation to $\mathrm{H}$ reduces to whether $\mathrm{E}_{2}$ provides positive incremental confirmation to $\mathrm{H}$ conditional on $\mathrm{E}_{1}$, i.e., $P(\mathrm{H} \mid$ $\left.\mathrm{E}_{1}, \mathrm{E}_{2}\right)>P\left(\mathrm{H} \mid \mathrm{E}_{1}\right)$. The answer is yes, and it makes no difference how strong the relationship between $H$ and $E_{2}$ is.

Proposition 3: If $\mathbf{E}=\left\{\mathrm{E}_{1}, \mathrm{E}_{2}\right\}$ and $\mathbf{E}^{\prime}=\left\{\mathrm{E}_{1}, \mathrm{E}_{3}\right\}$ are positive evidence sets for $\mathrm{H}$, then in any probability distribution $P\left(H, E_{1}, E_{2}, E_{3}\right\}$ that is Markov and Faithful to the causal graph in Figure 3, inc ${ }_{1}\left(\mathrm{H}, \mathrm{E}_{1}, \mathrm{E}_{2}\right)>$ inc $_{1}\left(\mathrm{H}, \mathrm{E}_{1}, \mathrm{E}_{3}\right){ }^{18}$

So coherence plays no role whatsoever in this case, except through the "side door." The coherence between $E_{1}$ and $E_{3}$ is entirely the result of the direct causal influence of $E_{1}$ on $E_{3}$. In the case of $E_{1}$ and $E_{2}$, however, positive coherence between $E_{1}$ and $E_{2}$ must arise through the path that goes through $\mathrm{H}$.

So in the case of $\mathbf{E}=\left\{\mathrm{E}_{1}, \mathrm{E}_{2}\right\}$ and $\mathbf{E}^{\prime}=\left\{\mathrm{E}_{1}, \mathrm{E}_{3}\right\}$, by the causal arrangements shown in Figure 3, and by the assumption that all evidence is individually positively relevant to $\mathrm{H}$, $\mathbf{E}$ will provide more confirmation than $\mathbf{E}$ ' to $\mathrm{H}$, regardless of the coherence of either set. ${ }^{19}$ It is the causal structure of the situation which drives things.

\section{Zero Coherence}

Now consider evidence sets $\mathbf{E}=\left\{\mathrm{E}_{1}, \mathrm{E}_{2}\right\}$ and $\mathbf{E} \boldsymbol{\prime}^{\prime}=\left\{\mathrm{E}_{1}, \mathrm{E}_{4}\right\}$. From the causal graph in Figure 3, we know that $\mathrm{E}_{1}$ and $\mathrm{E}_{4}$ are probabilistically independent, so $\mathbf{E}^{\text {'' has zero }}$ association and a Shogenji coherence equal to 1 . Is it nevertheless possible for $\mathbf{E}$ ', to provide more confirmation to $\mathrm{H}$ than $\mathbf{E}$, which has positive coherence? The answer, surprisingly, is yes.

Proposition $4:{ }^{20}$ In cases for which $\mathbf{E}$ and $\mathbf{E}$ ", are equally positive evidence (epe) sets for $\mathrm{H}$, then $\operatorname{inc}_{1}\left(\mathrm{H}, \mathrm{E}_{1}, \mathrm{E}_{4}\right)>\operatorname{inc}_{1}\left(\mathrm{H}, \mathrm{E}_{1}, \mathrm{E}_{2}\right)$ if and only if $S\left(\mathrm{E}_{1}, \mathrm{E}_{2}\right)>\frac{\alpha}{\beta}$

where $\alpha=\frac{P\left(\mathrm{H}_{i}\right)}{P(\mathrm{H})}$ and $\beta=\frac{P\left(\mathrm{H}_{1} \mathrm{E}_{1}, \mathrm{E}_{4}\right)}{P\left(\mathrm{H}_{\mathrm{E}}\right)}$.

The incremental confirmation from an evidence set with zero coherence (E', ) exceeds the confirmation from an evidence set with positive coherence $(\mathbf{E})$ just in case the coherence of $\mathbf{E}$ is greater than the ratio of the incremental confirmation provided by $E_{1}$ to the incremental confirmation provided by $\mathrm{E}_{4}$ after knowing $\mathrm{E}_{1}$. 


\section{Conclusion}

Explicating notions of coherence and confirmation have occupied philosophers of science for hundreds of years. Further, most every philosopher since William Whewell who has discussed both notions has connected them. Recently, many have tried to model these ideas and the connection between them using just the probability calculus. Attempts to connect coherence simpliciter to confirmation are bound to fail, as probabilistic models of coherence make no reference to either the reason for coherence or the reason any piece of evidence in a set of evidence should relate to H. In our view, any such effort ought to include, explicitly in the formalism, both the reason the evidence is coherent and how the evidence is connected to $\mathrm{H}$. We have tried to argue that focused correlation and causal structure move in this direction.

Focused correlation, a fully probabilistic measure which compares the coherence of an evidence set conditional on the hypothesis it might or might not confirm to the unconditional coherence of the set turns out to be a powerful indicator of when coherence helps confirmation as well as a powerful indicator of which evidence sets are more helpful than others. It is not the complete story, either, however, as in some cases comparing the focused correlation of two evidence sets underdetermines the confirmation relation.

Since evidence can be causally connected to other evidence and to the hypothesis in virtually any way possible, it turns out to be very useful to explicitly and formally model the causal structure governing the evidence and the hypothesis. Even when one connects causal structure to probability only qualitatively through independence and conditional independence, quite a lot about the relationship between coherence and confirmation can be adduced. In cases in which all the evidence are effects of the hypothesis and otherwise causally independent, coherence and confirmation are tightly connected. ${ }^{21}$ In cases in which the coherence between the evidence has nothing causally to do with the hypothesis, coherence and confirmation are utterly disconnected. In cases in which pieces of evidence are not caused by the hypothesis nor cause each other, the story is more complicated, but extremely rich nonetheless.

We have not offered a proof that focused correlation and/or causal structure are the only keys to the castle, nor do we think one is forthcoming. Nor have we offered anything approaching a complete theory of coherence and confirmation through focused correlation and causal structure. We hope, however, that we have shown that these tools can take us much farther than probabilistic models of coherence and confirmation alone. 


\section{References}

L. BonJour, The Structure of Empirical Knowledge. Cambridge, MA, Harvard University Press, 1985.

L. BonJour, The dialectics of foundationalism and coherentism, in J. Greco and E. Sosa (eds.), The Blackwell Guide to Epistemology. Malden, MA: Blackwell, 1999, 117-42.

L. BonJour, Epistemology. Rowman and Littlefield, Oxford, 2002.

L. Bovens, and S. Hartmann, Bayesian Epistemology. Oxford University Press, 2003a

L. Bovens, and S. Hartmann, Solving the Riddle of Coherence, Mind, 112, 2003b, 601633.

L. Bovens, and S. Hartmann, An impossibility result for coherence rankings. Philosophical Studies, 128, 2006, 77-91.

R. Carnap, The Logical Foundations of Probability. University of Chicago Press, 1962

L. J. Cohen, The Probable and the Provable, Oxford: Clarendon Press, 1977

C. B. Cross, Coherence and truth conducive justification. Analysis, 59(3), 1999, 186-93.

D. Danks and C. Glymour, Linearity properties of Bayes nets with binary variables. In J. Breese \& D. Koller (Eds.), Uncertainty in artificial intelligence: Proceedings of the 17th conference (UAI-2001) (pp. 98-104). San Francisco: Morgan Kaufmann, 2001

A. Dempster, Upper and lower probabilities induced by a multivalued mapping. Annals of Mathematical Statistics. 38, 1967, 325-339.

I. Douven and W. Meijs, Measuring coherence. Synthese, 156(3), 2007, 405-425.

J. Earman, Bayes or Bust: A Critical Examination of Bayesian Confirmation Theory. Cambridge, MA: MIT Press, 1992

E. Eells, and B. Fitelson, Symmetries and Asymmetries in Evidential Support. Philosophical Studies, 107(2), 2002, 129-142.

B. Fitelson, A probabilistic theory of coherence. Analysis, 63, 2003, 194-99. 
D. H. Glass, Coherence measures and their relations to fuzzy similarity and inconsistency in knowledge bases. Artificial Intelligence Review, 26, 2006, 227-249.

Clark.Glymour, What Went Wrong: Reflections on Science by Observation and The Bell Curve, Philosophy of Science, Vol. 65, No. 1 (Mar., 1998), 1-32.

Clark Glymour, R. Scheines, P. Spirtes, and K. Kelly, Discovering Causal Structure. Academic Press, 1987.

R. Haenni, J.W. Romeyn, G. Wheeler, G. and J. Williamson, Probabilistic Logic and Probabilistic Networks, Dordrecht: The Synthese Library, 2009

M. Huemer, Probability and coherence justification. Southern Journal of Philosophy, 35, 1997, 463-72.

R Jeffrey, The Logic of Decision. New York: McGraw-Hill, 1965

B. W. Junker and J.L. Ellis, A Characterization of Monotone Unidimensional Latent Variable Models. The Annals of Statistics, 25, 1997, 1327--1343.

P. Klein, and T. Warfield, What price coherence? Analysis, 54(3), 1994, 129-32.

H. Kyburg, Jr and M. Pittarelli, Set-Based Bayesianism. IEEE Transactions on Systems, Man and Cybernetics A, 26(3), 1996, 324-339.

I. Levi, The Enterprise of Knowledge. Cambridge, MA: MIT Press, 1980

C.I. Lewis, An Analysis of Knowledge and Valuation. Open Court, La Salle, 1946

W. Meijs, A corrective to Bovens and Hartmann's measure of coherence, Philosophical Studies, 133(2), 2004, 151-180.

W. Myrvold, Bayesianism and diverse evidence: a reply to Andrew Wayne. Philosophy of Science, 63, 1996, 661-5.

E. Olsson, What is the problem of coherence and truth? Journal of Philosophy, 94, 2002, 246-72.

E. Olsson, Against Coherence: Truth, Probability and Justification. Oxford University Press, Oxford, 2005

F. Ramsey, Truth and Probability, reprinted in Ramsey, 1931, The Foundations of Mathematics and other Logical Essays, Ch. VII, p.156-198, edited by R.B. Braithwaite, London: Kegan, Paul, Trench, Trubner \& Co., New York: Harcourt, 
Brace and Company. 1999 electronic edition, http://ideas.repec.org/h/hay/hetcha/ramsey1926.html

M. Schlosshauer and G. Wheeler, Focused correlation, confirmation, and the jigsaw puzzle of variable evidence, unpublished manuscript.

T. Shogenji, Is coherence truth conducive? Analysis, 59, 1999, 338-45.

R. Silva, C. Glymour, R Scheines, and P. Spirtes, Learning the Structure of Latent Linear Structure Models, Journal of Machine Learning Research, 7, 2006,191-246.

P. Spirtes, C. Glymour, and R. Scheines, Causation, Prediction, and Search. $2^{\text {nd }}$. edition. MIT Press, Cambridge, MA, 2000

G. Wheeler, Focused correlation and confirmation. The British Journal for the Philosophy of Science, 60(1), 2009, 79-100.

\section{G. Wheeler and R. Scheines, Coherence, Confirmation, and Causation, CMU Department of Philosophy Technical Report, Department of Philosophy, Carnegie Mellon, Pittsburgh, PA 15213, 2010.}

${ }^{1}$ Center for Artificial Intelligence, New University of Lisbon, Portugal, grw@fct.unl.pt
2 Department of Philosophy, Carnegie Mellon University, Pittsburgh, PA
${ }^{3}$ Models of coherence or confirmation, or the relation between them are discussed by Bovens and
Hartmann, 2003a,b, 2006; Douven, 2007; Fitelson, 2003; Glass, 2006; Meijs, 2004; Olsson, 2002,
Shogenji, 1999; Wheeler, 2009. Causal Bayes Nets, the probabilistic model of causal systems now
standard in computer science and statistics, are discussed in Pearl, 2000; and in Spirtes, Glymour, and Scheines, 2000.

${ }^{4}$ BonJour (1985, pp. 95-9).

${ }^{5}$ BonJour (2002, pp. viii, 202).

${ }^{6}$ See, for example, Huemer 1997, Cross 1999, Shogenji 1999, Bovens \& Hartmann 2003a, 2003b, 2005; Olsson 2002, 2005; Fitelson 2003; Meijs, 2004, Glass 2006.

${ }^{7}$ Other proposals along these lines have been made by Huemer 1997, Cross 1999, Olsson 2002, Fitelson 2003, Glass 2006, and Wheeler 2009.

${ }^{8}$ Although this definition of association is attributed to Shogenji in Bayesian epistemology, it predates him in the general statistics literature by several decades.

${ }^{9}$ Witness reports whose contents are logically related are not themselves logically related in this way, for it is perfectly possible to have a measure involving propositional variables $\mathrm{V}_{1}$ : witness 1 report $=$ The Butler did it, and $\mathrm{V}_{2}$ : witness 2 report $=$ The Butler did it or The Cook did it in which $\mathrm{V}_{1}$ and $\mathrm{V}_{2}$ are independent.

${ }^{10}$ Probability can be interpreted as credal or objective, we don't care. Our concern is only with how coherence, interpreted probabilistically, interacts with confirmation, also interpreted probabilistically.

${ }^{11}$ See Pearl, 2000, and Spirtes, Glymour, and Scheines, 2000.

${ }^{12}$ The Causal Markov Axiom applies to causally sufficient sets of variables.

${ }^{13}$ If $\mathrm{X}$ has no parents, then $\mathrm{P}(X \mid$ parents $(X))=\mathrm{P}(X)$.

${ }_{15}^{14}$ Again, the Faithfulness Axiom applies to causally sufficient sets of variables.

${ }^{15} A \|_{-} B \mid C$ is to be read: $A$ is independent of $B$ conditional on $C$, where $A, B$, and $C$ are random variables, or sets of random variables. If $A, B$, and $C$ are naturally interpreted as events, then they can just as easily be represented as a random variable with binary outcome, $0=$ the event did not occur, and $1=$ the event occurred. 
${ }^{16}$ This is because, in a singly connected Bayes net with binary variables, the correlation (which is proportional to the Shogenji association) between the evidence variables is simply the product of the correlation between the hypothesis and the evidence variables. See Danks and Glymour (2001).

${ }^{17}$ In the sense of Bovens and Hartmann (2006).

${ }^{18}$ The proof is given in Wheeler and Scheines (2010).

${ }^{19}$ The coherence between $\mathbf{E}$ ' could be as close to maximal as we like, and the coherence of $\mathbf{E}$ arbitrarily small (but positive), and it is nevertheless the case that $\mathbf{E}$ will provide more confirmation than $\mathbf{E}$ '.

${ }^{20}$ Again, the proof is given in Wheeler and Scheines (2010).

${ }^{21}$ Philosophers, statisticians, and computer scientists have learned a lot about how to tell, from data, whether or not a set of measured variables are indeed effects of an unmeasured common cause and otherwise causally independent, and so this case is epistemically particularly exciting. See Silva, Scheines, Glymour, and Spirtes (2006), Junker and Ellis (1997), and Glymour (1998). 